\title{
MODULAR DESCENT OF SIEGEL MODULAR FORMS OF HALF INTEGRAL WEIGHT AND AN ANALOGY OF THE MAASS RELATION
}

\author{
YOSHIO TANIGAWA
}

\section{Introduction}

In [8], H. Maass introduced the 'Spezialschar' which is now called the Maass space. It is defined by the relation of the Fourier coefficients of modular forms as follows. Let $f$ be a Siegel modular form on $S p(2, Z)$ of weight $k$, and let $f(Z)=\sum a(T) e(\operatorname{tr} T Z)$ be its Fourier expansion, where $e(z)=\exp (2 \pi i z)$ for $z \in C$. Then $f$ belongs to the Maass space if and only if

$$
a\left[\begin{array}{ll}
n, & \frac{r}{2} \\
\frac{r}{2}, & m
\end{array}\right)=\sum_{\substack{d \mid(n, r, m) \\
d>0}} d^{k-1} a\left(\begin{array}{cc}
\frac{n m}{d^{2}}, & \frac{r}{2 d} \\
\frac{r}{2 d}, & 1
\end{array}\right) .
$$

In a series of papers [8]-[10], he studied the connection with Jacobi forms of index 1 and the Dirichlet series of Andrianov type. In particular, using the Hecke operators acting on Jacobi forms, he showed that there is a lifting from the space of Jacobi forms of index 1 to the space of Siegel modular forms on $\operatorname{Sp}(2, Z)$. (See also D. Zagier [13] and M. Eichler and D. Zagier [2]).

On the other hand, Shimura's theory of Hecke operators on modular forms of half integral weight was generalized to Siegel modular forms by S. Hayakawa, K. Tanaka, T. Hina, and T. Ibukiyama. In this paper, we consider the forms of degree 2 and define an analogy of the Maass relation.

Let $f$ be a Siegel modular form on $\Gamma_{0}^{(2)}(N)$ of half integral weight $k / 2$ and character $\chi$ and let $f(Z)=\sum a(T) e(\operatorname{tr} T Z)$ be its Fourier expansion. We also write $a(T)=a(n, r, m)$ for $T=\left(\begin{array}{cc}n & r / 2 \\ r / 2 & m\end{array}\right)$. Then the analogy of the 
Maass relation can be written as

$$
\begin{aligned}
a\left(n, r, m p^{2}\right)= & a\left(n p^{2}, r, m\right) \\
& +\chi(p) \varepsilon_{p}^{k-1} p^{(k-3) / 2}\left(\left(\frac{n}{p}\right)-\left(\frac{m}{p}\right)\right) a\left(n, \frac{r}{p}, m\right) \\
& +\chi(p)^{2} p^{k-2}\left(a\left(\frac{n}{p^{2}}, \frac{r}{p^{2}}, m\right)-a\left(n, \frac{r}{p^{2}}, \frac{m}{p^{2}}\right)\right)
\end{aligned}
$$

for any prime number $p$ such that $(p, N)=1$. Here $\varepsilon_{d}=1$ if $d \equiv 1(\bmod$ 4) and $\varepsilon_{d}=i$ if $d \equiv 3(\bmod 4)$. We consider two kinds of Dirichlet series, namely

$$
\begin{aligned}
& \sum_{M \in \mathscr{R}} a(T[M])|\operatorname{det} M|^{-s}, \quad \text { where } \\
& \mathscr{R}=\left\{M \in M_{2}(Z) \cap G L(2, \boldsymbol{Q}) \mid(\operatorname{det} M, N)=1\right\} / G L(2, Z),
\end{aligned}
$$

and

$$
\sum_{\nu=0}^{\infty} a\left(p^{2 \nu+2 d} T\right) x^{\nu} \quad \text { for } T \not \equiv 0(\bmod p), \quad d \geqq 0 .
$$

We will show that if $f$ is a common eigenfunction of all the Hecke operators and $f$ satisfies the Maass relation, the denominators of (iii) and (iv) can be expressed by certain Dirichlet series of the form of integral weight of one variable associated to $f$.

I would like to express my thanks to S. Hayakawa for the useful discussion.

Notation and the definition of modular forms (G. Shimura [12], S. Hayakawa [3]).

Let $G^{(n)}$ be the symplectic group of degree $n$ and let $\bar{G}^{(n)}$ be the symplectic similitude group:

$$
\bar{G}^{(n)}=\left\{\left.\alpha \in G L(2 n, R)\right|^{t} \alpha I \alpha=\nu(\alpha) I, \nu(\alpha)>0\right\}
$$

where ${ }^{t} \alpha$ is the transpose of $\alpha$ and $I=\left(\begin{array}{cc}0 & 1_{n} \\ -1_{n} & 0\end{array}\right), 1_{n}=$ the unit matrix of order $n$. We denote the Siegel upper half plane by $\mathfrak{h}^{(n)}$. The group $\bar{G}^{(n)}$ acts on $\mathfrak{h}^{(n)}$ by

$$
\alpha(Z)=(A Z+B)(C Z+D)^{-1} \quad \text { for } \alpha=\left(\begin{array}{ll}
A & B \\
C & D
\end{array}\right) \in \bar{G}^{(n)} .
$$

Furthermore we define the group $\mathfrak{g}^{(n)}$ by 
$\mathfrak{g}^{(n)}=\left\{\xi=(\alpha, \varphi) \mid \begin{array}{l}\alpha \in \bar{G}^{(n)}, \varphi \text { : non zero holomorphic function on } \mathfrak{h}^{(n)} \\ \text { such that } \varphi(Z)^{2}=t \nu(\alpha)^{-n / 2} \operatorname{det}(C Z+D) \text { with } t \in C,|t|=1\end{array}\right\}$

with a law of multiplication as

$$
(\alpha, \varphi(Z))(\beta, \psi(Z))=(\alpha \beta, \varphi(\beta(Z)) \psi(Z)) .
$$

We denote the natural projection map by $P: \mathfrak{g}^{(n)} \rightarrow \bar{G}^{(n)}$.

Let $N$ be a positive integer divisible by 4 . Put

$$
\Gamma_{0}^{(n)}(N)=\left\{\left(\begin{array}{ll}
A & B \\
C & D
\end{array}\right) \in S p(n, Z) \mid C \equiv 0(\bmod N)\right\}
$$

which is called the congruence subgroup of level $N$, degree $n$. We define the theta series $\theta$ by

$$
\theta(Z)=\sum_{l \in Z^{n}} e\left({ }^{t} l Z l\right)
$$

where $e(z)=\exp (2 \pi i z)$. It is a holomorphic function on $\mathfrak{h}^{(n)}$ and has the following transformation formula;

$$
\begin{aligned}
& \theta(\alpha(Z))=J(\alpha, Z) \theta(Z) \\
& J(\alpha, Z)^{2}=\left(\frac{-1}{\operatorname{det} D}\right) \operatorname{det}(C Z+D) \quad \text { for } \alpha=\left(\begin{array}{ll}
A & B \\
C & D
\end{array}\right) \in \Gamma_{0}^{(n)}(4) .
\end{aligned}
$$

$J(\alpha, Z)$ is called the theta-multiplier system. Let $L$ be an imbedding of $\Gamma_{0}^{(n)}(4)$ to $\mathrm{g}^{(n)}$ defined by

$$
L(\alpha)=(\alpha, J(\alpha, Z)) .
$$

We denote the image of $\Gamma_{0}^{(n)}(N)$ under $L$ by $\Delta_{0}^{(n)}(N)$.

For any function $f$ on $\mathfrak{h}^{(n)}$ and any integer $k$, we define an action of $\xi=(\alpha, \varphi)$ by $\left(f \mid[\xi]_{k}\right)(Z)=\varphi(Z)^{-k} f(\alpha(Z))$. It is easily seen that $f \mid[\xi \eta]_{k}=$ $\left(f \mid[\xi]_{k}\right) \mid[\eta]_{k}$.

From now on, let $k$ be an odd integer and $\chi$ a Dirichlet character modulo $N$.

Definition. A holomorphic function $f$ on $\mathfrak{h}^{(n)}$ is called a Siegel modular form on $\Gamma_{0}^{(n)}(N)$ of weight $k / 2$ and character $\chi$ if and only if

(i ) $f \mid[\xi]_{k}=\chi(\operatorname{det} D) f$ for any $\xi \in \Delta_{0}^{(n)}(N), P(\xi)=\left(\begin{array}{ll}* & * \\ * & D\end{array}\right)$,

(ii) if $n=1, f \mid[\xi]_{k}$ is bounded on $\left\{Z \in \mathfrak{h}^{(n)} \mid \operatorname{Im} Z>\varepsilon 1_{n}\right\}$ for any $\xi \in P^{-1}(S p(n, Z))$ and any positive real number $\varepsilon$. 
The space of all such forms is denoted by $G_{k / 2}\left(\Gamma_{0}^{(n)}(N), \chi\right)$. Furthermore $f \in G_{k / 2}\left(\Gamma_{0}^{(n)}(N), \chi\right)$ is called a cusp form if

(iii) $\Phi\left(f \mid[\xi]_{k}\right)=0$ for any $\xi \in P^{-1}(\operatorname{Sp}(n, Z))$,

where $\Phi$ is the Siegel operator. The space of all cusp forms is denoted by $S_{k / 2}\left(\Gamma_{0}^{(n)}(N), \chi\right)$.

We also use the following notation;

$$
\varepsilon_{d}= \begin{cases}1 & \text { if } d \equiv 1(\bmod 4) \\ i & \text { if } d \equiv 3(\bmod 4)\end{cases}
$$

$(-)=$ the Legendre symbol in the sense of G. Shimura [12],

$$
\begin{aligned}
& \sqrt{z}=|z|^{1 / 2} \exp \left(\frac{1}{2} \arg z\right), \text { with } \quad-\pi<\arg z \leqq \pi, \\
& z^{k / 2}=(\sqrt{z})^{k} \quad \text { for any } k \in Z .
\end{aligned}
$$

When $n=1$, we always omit the superscript (1). For matrices $M$ and $L, M[L]={ }^{t} L M L$.

\section{§ 1. Jacobi forms of half integral weight}

As above, let $N$ be a positive integer divisible by $4, \chi$ a Dirichlet character modulo $N$ and $k$ an odd positive integer. Let $\Gamma_{0}^{(2)}(N)$ be a congruence subgroup of level $N$, degree 2 and $\Gamma_{0}(N)$ be an usual elliptic congruence subgroup of level $N$. Let $f$ be a Siegel modular form of degree 2 on $\Gamma_{0}^{(2)}(N)$ of weight $k / 2$ and character $\chi$. $f$ has a Fourier expansion

$$
f(Z)=\sum_{T} a(T) e(\operatorname{tr} T Z)
$$

where $T$ runs over the set $\Lambda_{2}$ of half integral semi positive definite symmetric matrices. We also write $a(T)=a(n, r, m)$ for $T=\left(\begin{array}{cc}n & r / 2 \\ r / 2 & m\end{array}\right) \in \Lambda_{2}$. If $Z=\left(\begin{array}{cc}\tau & z \\ z & \tau^{\prime}\end{array}\right)$, then $\operatorname{tr}(T Z)=n \tau+r z+m \tau^{\prime}$. Therefore, rearranging the expansion (1.1) with respect to $\tau^{\prime}$, we get

$$
f(Z)=\sum_{m \geqq 0} \phi_{m, f}(\tau, z) e\left(m \tau^{\prime}\right)
$$

where

$$
\phi_{m, f}(\tau, z)=\sum_{\substack{n \geq 0, r \\ 4 m n \geqq r^{2}}} a(n, r, m) e(n \tau+r z) .
$$

For any pair of rational integers $\lambda, \mu$, we put 


$$
U_{\lambda, \mu}=\left(\begin{array}{rr|rr}
1 & 0 & 0 & \mu \\
\lambda & 1 & \mu & 0 \\
\hline 0 & 1 & -\lambda \\
0 & 0 & 1
\end{array}\right)
$$

This belongs to $\Gamma_{0}^{(2)}(N)$, so we have

$$
f\left(U_{\lambda, \mu}(Z)\right)=J\left(U_{\lambda, \mu}, Z\right)^{k} f(Z)=f(Z) .
$$

This implies that

$$
\phi_{m, f}(\tau, z+\lambda \tau+\mu)=e\left(-m\left(\lambda^{2} \tau+2 \lambda z\right)\right) \phi_{m, f}(\tau, z) .
$$

On the other hand, for any $\gamma=\left(\begin{array}{ll}a & b \\ c & d\end{array}\right) \in \Gamma_{0}(N)$, we put

$$
M_{r}=\left(\begin{array}{ll|ll}
a & 0 & b & 0 \\
0 & 1 & 0 & 0 \\
\hline c & 0 & d & 0 \\
0 & 0 & 0 & 1
\end{array}\right),
$$

which also belongs to $\Gamma_{0}^{(2)}(N)$. Then, by noting that

$$
J\left(M_{r}, Z\right)=\left(\frac{c}{d}\right) \varepsilon_{d}^{-1} \sqrt{c \tau+d}
$$

we get

$$
\phi_{m, f}\left(\frac{a \tau+b}{c \tau+d}, \frac{z}{c \tau+d}\right)=\chi(d)\left(\frac{c}{d}\right) \varepsilon_{d}^{-k} \sqrt{c \tau+d^{k}} e\left(\frac{m c z^{2}}{c \tau+d}\right) \phi_{m, f}(\tau, z) .
$$

Now we set the following definition.

Definition 1. A holomorphic function $\phi(\tau, z)$ on $\mathfrak{h} \times \boldsymbol{C}$ is called a Jacobi form on $\Gamma_{0}(N)$ of weight $k / 2$, index $m$ and character $\chi$ if it satisfies the following conditions:

(i ) $\phi(\tau, z+\lambda \tau+\mu)=e\left(-m\left(\lambda^{2} \tau+2 \lambda z\right)\right) \phi(\tau, z)$ for any $(\lambda, \mu) \in Z^{2}$,

(ii) $\phi\left(\frac{a \tau+b}{c \tau+d}, \frac{z}{c \tau+d}\right)=\chi(d)\left(\frac{c}{d}\right) \varepsilon_{d}^{-k} \sqrt{c \tau+d^{k}} e\left(\frac{m c z^{2}}{c \tau+d}\right) \phi(\tau, z)$ for any $\left(\begin{array}{ll}a & b \\ c & d\end{array}\right) \in \Gamma_{0}(N)$

(iii) for each $\sigma=\left(\begin{array}{ll}a & b \\ c & d\end{array}\right) \in S L(2, Z)$, there is an integer $d_{\sigma}$ such that the function defined by 


$$
\phi^{\sigma}(\tau, z)=\phi\left(\frac{a \tau+b}{c \tau+b}, \frac{z}{c \tau+d}\right) \sqrt{c \tau+d^{-k}} e\left(-\frac{m c z^{2}}{c \tau+d}\right)
$$

has a Fourier expansion of the form

$$
\sum_{n, r \in\left(1 / d_{\sigma) Z}\right), 4 m n \geqq r^{2}} a^{\sigma}(n, r) e(n \tau+r z) .
$$

The space of all Jacobi forms is denoted by $J_{k / 2, m}\left(\Gamma_{0}(N), \chi\right)$. If $\phi \epsilon$ $J_{k / 2, m}\left(\Gamma_{0}(N), \chi\right)$ satisfies the further condition:

(iv) $a^{\sigma}(n, r)=0$ if $4 m n=r^{2}$,

it is called a (Jacobi's) cusp form, and we denote the space of all cusp forms by $J_{k / 2, m}^{0}\left(\Gamma_{0}(N), \chi\right)$.

By the above argument we get the following proposition.

Proposition 1. Let $f$ be a Siegel modular form on $\Gamma_{0}^{(2)}(N)$ of weight $k / 2$ and character $\chi$. For each $m \geqq 0$, define a function $\phi_{m, f}(\tau, z)$ on $\mathfrak{h} \times \boldsymbol{C}$ by

$$
\phi_{m, f}(\tau, z)=\sum_{\substack{n \geq 0, r \\ 4 m n \geqq r^{2}}} a(n, r, m) e(n \tau+r z) .
$$

Then $\phi_{m, f}(\tau, z)$ is a Jacobi form on $\Gamma_{0}(N)$ of weight $k / 2$, index $m$ and character $\chi$. Furthermore if $f$ is a cusp form, then $\phi_{m, f}$ is also a cusp form for each $m$.

The condition (iii) or (iv) is clear by the definition of Siegel modular forms.

\section{§2. Transformation formula of $c_{\alpha}(\tau)$}

We suppose that $m$ is a positive integer. As is well known, a function satisfying (i) of the Definition 1 , is a theta function. In fact, the following theta functions

$$
\begin{aligned}
\Theta_{\alpha}(\tau, z) & =\theta_{\alpha, 0}(2 m \tau, 2 m z) \\
& =\sum_{l \in \boldsymbol{Z}} e\left((l+\alpha)^{2} m \tau+2(l+\alpha) m z\right), \quad \text { for } \alpha \in \frac{1}{2 m} Z / Z
\end{aligned}
$$

form a basis of the space of such functions (cf. J. Igusa [6]). Therefore we have

$$
\phi(\tau, z)=\sum_{\alpha \in(1 / 2 m) Z / Z} c_{\alpha}(\tau) \Theta_{\alpha}(\tau, z)
$$

for any Jacobi form $\phi(\tau, z) \in J_{k / 2, m}\left(\Gamma_{0}(N), \chi\right)$. In particular, if $\phi_{m, f}(\tau, z)$ is 
obtained from $f \in G_{k / 2}\left(\Gamma_{0}^{(2)}(N), \chi\right)$ by Proposition 1, we have

$$
\phi_{m, f}(\tau, z)=\sum_{\alpha \in(1 / 2 m) Z / Z} c_{\alpha, f}(\tau) \Theta_{\alpha}(\tau, z)
$$

with

$$
c_{\alpha, f}(\tau)=\sum_{\substack{n \geq 0 \\ 4 m n \geqq r^{2}}} a(n, r, m) e\left(\left(n-\frac{r^{2}}{4 m}\right) \tau\right)
$$

for $\alpha=r / 2 m$.

We study the transformation formula of $c_{\alpha}(\tau)$ under $\Gamma_{0}(N)$. It is also well-known that there are constants $u_{\alpha \beta}(\sigma)$ which do not depend on $\tau$ and $z$, and

$$
\Theta_{\alpha}\left(\frac{a \tau+b}{c \tau+d}, \frac{z}{c \tau+d}\right)=e\left(\frac{m c z^{2}}{c \tau+d}\right)(c \tau+d)^{1 / 2} \sum_{\beta} u_{\alpha \beta}(\sigma) \Theta_{\beta}(\tau, z)
$$

for any $\sigma=\left(\begin{array}{ll}a & b \\ c & d\end{array}\right) \in S L(2, Z)$. The matrix $\left(u_{\alpha \beta}(\sigma)\right)$ is unitary (cf. J. Igusa [6]).

LEMMA 1. The functions $c_{\alpha}(\tau)$ in (2.2) satisfy the following transformation formula:

$$
\chi(d)\left(\frac{c}{d}\right) \varepsilon_{d}^{-k}(c \tau+d)^{(k-1) / 2} c_{\beta}(\tau)=\sum_{\alpha} u_{\alpha \beta}(\sigma) c_{\alpha}\left(\frac{a \tau+b}{c \tau+d}\right)
$$

for any $\sigma=\left(\begin{array}{ll}a & b \\ c & d\end{array}\right) \in \Gamma_{0}(N)$

Proof. It is easily proved by (ii) of Definition 1, (2.2) and (2.5).

By changing the variable, (2.6) is equivalent to

$$
c_{\beta}\left(\frac{a \tau+b}{c \tau+d}\right)=\chi(d)\left(\frac{-c}{a}\right) \varepsilon_{a}^{k}(c \tau+d)^{(k-1) / 2} \sum_{\alpha} u_{\alpha \beta}\left(\sigma^{-1}\right) c_{\alpha}(\tau) .
$$

Lemma 2. Let $\omega=\left(\begin{array}{rr}0 & -1 \\ 1 & 0\end{array}\right)$. Then

$$
u_{\alpha \beta}(\omega)=\frac{1}{\sqrt{2 m}} \exp \left(-\frac{\pi i}{4}\right) e(-2 m \alpha \beta) .
$$

Proof. We recall the well-known theta formula:

$$
\sum_{\xi \in Z} e\left(\frac{1}{2}(x+\xi)^{2} Z+w(x+\xi)\right)=(-i Z)^{-1 / 2} \sum_{\xi \in Z} e\left(-\frac{1}{2}(w-\xi)^{2} Z^{-1}+\xi x\right)
$$

for $w \in C, x \in R$, and $Z \in \mathfrak{h}$. We put $x=\alpha, Z=-2 m / \tau$, and $w=2 m z / \tau$. 
Then the left hand side is equal to $\Theta_{\alpha}(-1 / \tau, z / \tau)$. On the other hand, the right hand side is equal to

$$
\left(\frac{\tau}{2 i m}\right)^{1 / 2} e\left(\frac{m z^{2}}{\tau}\right) \sum_{\xi \in Z} e\left(-z \xi+\frac{\tau \xi^{2}}{4 m}+\alpha \xi\right) .
$$

Put $\eta=-\xi / 2 m$. Then the above sum becomes

$$
\begin{aligned}
\sum_{\eta} e & \left(2 m z \eta+m \tau \eta^{2}-2 m \alpha \eta\right) \\
& =\sum_{\beta \in(1 / 2 m) Z / Z, l \in Z} e\left(2 m z(\beta+l)+m \tau(\beta+l)^{2}-2 m \alpha(\beta+l)\right) \\
& =\sum_{\beta \in(1 / 2 m) Z / Z} e(-2 m \alpha \beta) \Theta_{\beta}(\tau, z) .
\end{aligned}
$$

This completes the proof.

LEMma 3. The following formulas hold for $c_{\alpha}(\tau)$.

(i ) $c_{-\alpha}(\tau)=\chi(-1) c_{\alpha}(\tau)$,

(ii) $c_{\alpha}(\tau+b) e\left(\alpha^{2} m b\right)=c_{\alpha}(\tau)$ for any $b \in Z$,

(iii) $(N \tau+1)^{(k-1) / 2} c_{\beta}(\tau)=\sum_{\alpha} \xi_{\alpha, \beta} c_{\alpha}\left(\frac{\tau}{N \tau+1}\right)$

where $\xi_{\alpha, \beta}=\frac{1}{2 m} \sum_{\gamma \in(1 / 2 m) Z / Z} e\left(-N \gamma^{2} m\right) e(2 m \gamma(\beta-\alpha))$.

Proof. These formulas are derived from (2.6) by the concrete values of $u_{\alpha \beta}\left(\left(\begin{array}{ll}1 & b \\ 0 & 1\end{array}\right)\right), u_{\alpha \beta}\left(-1_{2}\right)$ and $u_{\alpha \beta}\left(\left(\begin{array}{ll}1 & 0 \\ N & 1\end{array}\right)\right)$. In fact, it is easily seen from (2.1) that

$$
u_{\alpha \beta}\left(\left(\begin{array}{ll}
1 & b \\
0 & 1
\end{array}\right)\right)= \begin{cases}e\left(\alpha^{2} m b\right) & \text { if } \alpha=\beta \\
0 & \text { if } \alpha \neq \beta\end{cases}
$$

for $b \in Z$, and

$$
u_{\alpha \beta}\left(-1_{2}\right)= \begin{cases}1 & \text { if } \alpha=-\beta \\ 0 & \text { if } \alpha \neq-\beta .\end{cases}
$$

We get (i) and (ii) from these. In order to compute the value $u_{\alpha \beta}(\sigma)$, $\sigma=\left(\begin{array}{ll}1 & 0 \\ N & 1\end{array}\right)$, we note the decomposition of $\sigma: \sigma=\omega\left(\begin{array}{rr}-1 & N \\ 0 & -1\end{array}\right) \omega . \quad$ If we put $\tau^{\prime}=\omega(\tau), w=-z / \tau$, we get

$\Theta_{\alpha}\left(\frac{\tau}{N \tau+1}, \frac{z}{N \tau+1}\right)=\Theta_{\alpha}\left(-\frac{1}{\tau^{\prime}-N}, \frac{w}{\tau^{\prime}-N}\right)$ 


$$
\begin{aligned}
= & e\left(\frac{m w^{2}}{\tau^{\prime}-N}\right)\left(\tau^{\prime}-N\right)^{1 / 2} \sum_{\gamma} u_{\alpha \gamma}(\omega) \Theta_{\gamma}\left(\tau^{\prime}-N, w\right) \\
= & e\left(-\frac{m z^{2}}{\tau(N \tau+1)}\right)\left(-\frac{1}{\tau}-N\right)^{1 / 2} \sum_{\gamma} u_{\alpha \gamma}(\omega) e\left(-N \gamma^{2} m\right) \Theta_{-r}\left(-\frac{1}{\tau}, \frac{z}{\tau}\right) \\
= & e\left(-\frac{m z^{2}}{\tau(N \tau+1)}\right) e\left(\frac{m z^{2}}{\tau}\right)\left(-\frac{1}{\tau}-N\right)^{1 / 2} \tau^{1 / 2} \\
& \times \sum_{\gamma, \beta} e\left(-N \gamma^{2} m\right) u_{\alpha \gamma}(\omega) u_{-\gamma, \beta}(\omega) \Theta_{\beta}(\tau, z) .
\end{aligned}
$$

By noting that $(-1 / \tau-N)^{1 / 2} \tau^{1 / 2}=i(N \tau+1)^{1 / 2}$, we have

$$
\begin{aligned}
u_{\alpha \beta}(\sigma) & =i \sum_{\gamma} e\left(-N \gamma^{2} m\right) u_{\alpha \gamma}(\omega) u_{-\gamma, \beta}(\omega) \\
& =\frac{1}{2 m} \sum_{\gamma} e\left(-N \gamma^{2} m\right) e(2 m \gamma(\beta-\alpha)) .
\end{aligned}
$$

\section{§ 3. Modular descent of Jacobi forms of index 1}

Throughout this section we will consider the case $m=1$. A Jacobi form $\phi \in J_{k / 2,1}\left(\Gamma_{0}(N), \chi\right)$ is expressed as

$$
\phi(\tau, z)=c_{0}(\tau) \Theta_{0}(\tau, z)+c_{1 / 2}(\tau) \Theta_{1 / 2}(\tau, z) .
$$

THEOREM 1. Let the notation be as above. Let $\chi_{1}$ be a character modulo $N$ defined by $\chi_{1}(m)=\chi(m)\left(\frac{-1}{m}\right)^{(k-1) / 2}$. Then $c_{0}(\tau)$ is an elliptic modular form on $\Gamma_{0}(N)$ of weight $(k-1) / 2$ and character $\chi_{1}$. Furthermore if $\phi(\tau, z)$ is a cusp form, $c_{0}(\tau)$ is also a cusp form.

Proof. First we deal with the case $N=4$. We have $\xi_{0,0}=1, \xi_{1 / 2,0}=0$ where $\xi_{\alpha, \beta}$ is defined in Lemma 3. Therefore, by the same Lemma, we have

$$
c_{0}(\tau+1)=c_{0}(\tau) \quad \text { and } \quad c_{0}\left(\frac{\tau}{4 \tau+1}\right)=(4 \tau+1)^{(k-1) / 2} c_{0}(\tau)
$$

It is well known that $\Gamma_{0}(4)$ is generated by $\left(\begin{array}{ll}1 & 1 \\ 0 & 1\end{array}\right)$ and $\left(\begin{array}{ll}1 & 0 \\ 4 & 1\end{array}\right)$. Hence $c_{0}(\tau)$ is a modular form on $\Gamma_{0}(4)$ of weight $(k-1) / 2$ and trivial character $\chi_{0}$.

Before proving the general case, we note the following remark. There exists a Jacobi form such that $c_{0}(\tau)$ and $c_{1}(\tau)$ are not identically zero. In fact, let $M$ be an even unimodular quadratic lattice of dimension 8 (cf. O.T. O'Meara [11]) and let $L=M \perp\langle 2\rangle$. Then the theta series 


$$
f(Z)=\vartheta(Z, L)=\sum_{P \in M_{9},{ }_{2}(Z)} e\left(\frac{1}{2} \operatorname{tr}(L[P] Z)\right)
$$

is contained in $G_{9 / 2}\left(\Gamma_{0}^{(2)}(4), \chi_{0}\right)$ and the Jacobi form of index 1 obtained from $f(Z)$ gives such an example, i.e. $c_{0, f}(\tau)$ and $c_{1 / 2, f}(\tau)$ are not identically zero. By (2.7) and the fact just proved above, we have two relations of $c_{0, f}$ and $c_{1 / 2, f}$ :

$$
\begin{aligned}
& c_{0, f}(\sigma(\tau))=\left(\frac{-c}{a}\right) \varepsilon_{a}(c \tau+d)^{(k-1) / 2}\left(u_{0,0}\left(\sigma^{-1}\right) c_{0, f}(\tau)+u_{1 / 2, j}\left(\sigma^{-1}\right) c_{1 / 2, f}(\tau)\right), \\
& c_{0, f}(\sigma(\tau))=(c \tau+d)^{(k-1) / 2} c_{0, f}(\tau),
\end{aligned}
$$

for any $\sigma=\left(\begin{array}{ll}a & b \\ c & d\end{array}\right) \in \Gamma_{0}(4)$. But the $u_{\alpha \beta}\left(\sigma^{-1}\right)$ are the quantities relating only to the theta series and do not depend on a Jacobi form. Therefore we have

$$
\begin{aligned}
& \left(\frac{-c}{a}\right) \varepsilon_{a} u_{0,0}\left(\sigma^{-1}\right)=1 \\
& u_{1 / 2,0}\left(\sigma^{-1}\right)=0 .
\end{aligned}
$$

Now let $N, k$ and $\chi$ be general. We have, by (2.7) and (3.1),

$$
c_{0}\left(\frac{a \tau+b}{c \tau+d}\right)=\chi_{1}(d)(c \tau+d)^{(k-1) / 2} c_{0}(\tau) .
$$

Since (2.5) holds for any $\sigma=\left(\begin{array}{ll}a & b \\ c & d\end{array}\right) \in S L(2, Z)$, we have

$$
\begin{array}{r}
\phi\left(\frac{a \tau+b}{c \tau+d}, \frac{z}{c \tau+d}\right) \sqrt{c \tau+d^{-k}} e\left(-\frac{c z^{2}}{c \tau+d}\right) \\
=\sum_{\alpha} c_{\alpha}\left(\frac{a \tau+b}{c \tau+d}\right)(c \tau+d)^{-(k-1) / 2} \sum_{\beta} u_{\alpha \beta}(\sigma) \Theta_{\beta}(\tau, z) .
\end{array}
$$

Hence

$$
\sum a^{\sigma}(n, r) e(n \tau+r z)=\sum_{\beta}\left(\sum_{\alpha} u_{\alpha \beta}(\sigma)\left(c_{\alpha} \mid[\sigma]\right)(\tau)\right) \Theta_{\beta}(\tau, z),
$$

where $\left(c_{\alpha} \mid[\sigma]\right)(\tau)=c_{\alpha}\left(\frac{a \tau+b}{c \tau+d}\right)(c \tau+d)^{-(k-1) / 2}$. Comparing the both sides of the above formula, we have

$$
a^{\sigma}(n, r)=0 \quad \text { if } r \notin Z
$$

and 


$$
\sum_{\alpha} u_{\alpha \beta}(\sigma)\left(c_{\alpha} \mid[\sigma]\right)(\tau)=\sum_{\substack{n \\ 4 n \geq r^{2}}} a^{\sigma}(n, r) e\left(\left(n-\frac{r^{2}}{4}\right) \tau\right)
$$

for $\beta=r / 2$. If we put $\left(u_{\alpha \beta}(\sigma)\right)^{-1}=\left(u_{\alpha \beta}^{\prime}(\alpha)\right)$, then we get

$$
\left(c_{\alpha} \mid[\sigma]\right)(\tau)=\sum_{\substack{r=0 \\ 4 n-r^{2} \geqq 0}}^{1} \sum_{n} u_{\alpha \beta}^{\prime}(\sigma) a^{\sigma}(n, r) e\left(\left(n-\frac{r^{2}}{4}\right) \tau\right),
$$

thus we obtain our assertion.

Next we consider the function $c_{1 / 2}(\tau)$. Let $\Gamma_{0}(N, M)$ be the subgroup of $\Gamma_{0}(N)$ defined by

$$
\Gamma_{0}(N, M)=\left\{\left(\begin{array}{ll}
a & b \\
c & d
\end{array}\right) \in S L(2, Z) \mid c \equiv 0(\bmod N), b \equiv 0(\bmod M)\right\} .
$$

Lemma 4. Put $\eta_{0}=\left(\begin{array}{ll}1 & 1 \\ 0 & 1\end{array}\right)$ and $\sigma_{0}=\left(\begin{array}{ll}1 & 0 \\ 4 & 1\end{array}\right)$. Let $\rho$ be an element of $\Gamma_{0}(4)$ and let

$$
\rho=\eta_{0}^{\ell_{1}} \sigma_{0}^{m_{1}} \cdots \eta_{0}^{\ell_{t}} \sigma_{0}^{m_{t}}, \quad \ell_{i}, m_{i} \in Z
$$

be its expression as the products of $\eta_{0}$ and $\sigma_{0}$. Then $\rho$ belongs to $\Gamma_{0}(4,4)$ if and only if

$$
\sum_{i=1}^{t} \ell_{i} \equiv 0(\bmod 4)
$$

The proof of this lemma is easy and we omit it here.

THEOREM 2. Let the notation and the assumptions be as in Theorem 1. Then $c_{1 / 2}(\tau)$ is an elliptic modular form on $\Gamma_{0}(N, 4)$ of weight $(k-1) / 2$ and character $\chi_{1}$.

This theorem is proved by the same way as Theorem 1 .

ExAmple. Let $k=3$ and let $A=\left(\begin{array}{ccc}2 a & f & e \\ f & 2 b & d \\ e & d & 2 c\end{array}\right)$ be an even ternary quadratic form. We also write $A=[a, b, c, d, e, f]$ for simplicity. The theta series corresponding to $A$ is defined by

$$
\theta(Z ; A)=\sum_{P \in M_{3}, 2} e\left(\frac{1}{2} \operatorname{tr}(A[P] Z)\right)
$$

By Theorem 1, the descended form $c_{0}(\tau ; A)$ belongs to $G_{1}\left(\Gamma_{0}(N),(-2 \operatorname{det} A)\right)$, $N=$ the level of $A$, and has a Fourier expansion 


$$
c_{0}(\tau ; A)=\sum_{n \geq 0} \#\left\{P \in M_{3,2}(Z) \mid \frac{1}{2} A[P]=\left(\begin{array}{cc}
n & 0 \\
0 & 1
\end{array}\right)\right\} e(n \tau) .
$$

Let $\eta(\tau)$ be the Dedekind eta function. Then we have

$$
\begin{aligned}
& \eta(\tau) \eta(23 \tau)=\frac{1}{8} c_{0}(\tau ;[1,1,6,0,-1,0])-\frac{1}{4} c_{0}(\tau ;[1,4,24,-4,0,0]) \\
& +\frac{1}{2} c_{0}(\tau ;[1,8,12,-4,0,0])-\frac{1}{4} c_{0}(\tau ;[1,3,18,-2,0,0]) \\
& -\frac{1}{4} c_{0}(\tau ;[1,2,12,-2,0,0]) \quad \in S_{1}\left(\Gamma_{0}\left(2^{2} \cdot 23\right),(-23)\right), \\
& \eta(2 \tau) \eta(22 \tau)=\frac{1}{8} c_{0}(\tau ;[1,1,11,0,0,0])-\frac{1}{12} c_{0}(\tau ;[1,1,4,0,1,1]) \\
& \in S_{1}\left(\Gamma_{0}\left(2^{2} \cdot 11\right),(-11)\right) \\
& \eta(3 \tau) \eta(21 \tau)=\frac{1}{8} c_{0}(\tau ;[1,1,16,0,-1,0])-\frac{1}{4} c_{0}(\tau ;[1,4,4,-1,0,0]) \\
& +\frac{1}{4} c_{0}(\tau ;[1,4,16,-2,0,0])-\frac{1}{4} c_{0}(\tau ;[1,4,64,-4,0,0]) \\
& \in S_{1}\left(\Gamma_{0}\left(2^{2} \cdot 3^{2} \cdot 7\right),(-7)\right), \\
& \eta(4 \tau) \eta(20 \tau)=\frac{1}{8} c_{0}(\tau:[1,1,20,0,0,0)]-\frac{1}{4} c_{0}(\tau ;[1,4,5,0,0,0]) \\
& \in S_{1}\left(\Gamma_{0}\left(2^{4} \cdot 5\right),(-5)\right) \\
& \eta(5 \tau) \eta(19 \tau)=\frac{1}{4} c_{0}(\tau ;[1,1,24,0,-1,0])-\frac{1}{4} c_{0}(\tau ;[1,4,6,-1,0,0]) \\
& -\frac{1}{8} c_{0}(\tau ;[1,1,95,0,0,0]) \quad \in S_{1}\left(\Gamma_{0}\left(2^{2} \cdot 5 \cdot 19\right),(-95)\right), \\
& \eta(6 \tau) \eta(18 \tau)=\frac{1}{8} c_{0}(\tau ;[1,1,27,0,0,0])-\frac{1}{4} c_{0}(\tau ;[1,2,4,-1,0,-1]) \\
& \in S_{1}\left(\Gamma_{0}\left(2^{2} \cdot 3^{3}\right)\right. \\
& \eta(7 \tau) \eta(17 \tau)=\frac{1}{4} c_{n}(\tau ;[1,1,30,0,-1,0])-\frac{1}{4} c_{0}(\tau ;[1,4,8,-3,0,0]) \\
& -\frac{1}{8} c_{0}(\tau ;[1,1,119,0,0,0]) \quad \in S_{1}\left(\Gamma_{0}\left(2^{2} \cdot 7 \cdot 17\right),(-119)\right),
\end{aligned}
$$




$$
\begin{aligned}
& \eta(8 \tau) \eta(16 \tau)=\frac{1}{8} c_{0}(\tau ;[1,1,32,0,0,0])-\frac{1}{4} c_{0}(\tau ;[1,4,9,4,0,0]) \\
& \in S_{1}\left(\Gamma_{0}\left(2^{7}\right),(-2)\right) \\
& \eta(9 \tau) \eta(15 \tau)=\frac{1}{4} c_{0}(\tau ;[1,1,34,0,-1,0])-\frac{1}{4} c_{0}(\tau ;[1,4,9,-3,0,0]) \\
& -\frac{1}{8} c_{0}(\tau ;[1,1,135,0,0,0]) \quad \in S_{1}\left(\Gamma_{0}\left(2^{2} \cdot 3^{3} \cdot 5\right),(-15)\right), \\
& \eta(10 \tau) \eta(14 \tau)=\frac{1}{8} c_{0}(\tau ;[1,1,35,0,0,0])-\frac{1}{4} c_{0}(\tau ;[1,3,4,3,1,1]) \\
& \in S_{1}\left(\Gamma_{0}\left(2^{2} \cdot 5 \cdot 7\right),(-35)\right) \\
& \eta(11 \tau) \eta(13 \tau)=\frac{1}{4} c_{0}(\tau ;[1,1,36,0,-1,0])-\frac{1}{4} c_{0}(\tau ;[1,4,9,-1,0,0]) \\
& -\frac{1}{8} c_{0}(\tau ;[1,1,143,0,0,0]) \in S_{1}\left(\Gamma_{0}\left(2^{2} \cdot 11 \cdot 13\right),(-143)\right), \\
& \eta(12 \tau) \eta(12 \tau)=\frac{1}{8} c_{0}(\tau ;[1,1,36,0,0,0])-\frac{1}{4} c_{0}(\tau ;[1,4,9,0,0,0]) \\
& \in S_{1}\left(\Gamma_{0}\left(2^{4} \cdot 3^{2}\right), 1\right)
\end{aligned}
$$

The above expressions are not unique.

\section{§4. Review of Hecke operators}

Let $n$ be a positive integer. Let $\Delta^{(n)}=\Delta_{0}^{(n)}(N)$ and $\tilde{\Delta}^{(n)}$ the commensurator of $\Delta^{(n)}$ in $\mathfrak{g}^{(n)}$. We define two semigroups of $\tilde{\Delta}^{(n)}$ by

$$
\begin{gathered}
\bar{S}_{0}^{(n)}=\left\{\xi=(\alpha, \varphi) \in \tilde{\Delta}^{(n)} \mid \alpha=\left(\begin{array}{ll}
A & B \\
C & D
\end{array}\right) \in \bar{G}^{(n)} \cap M_{2 n}(Z),\right. \\
C \equiv 0(\bmod N),(\nu(\alpha), N)=1\} \\
\bar{S}^{(n)}=\left\{\xi=(\alpha, \varphi) \in \bar{S}_{0}^{(n)} \mid \nu(\alpha) \text { is square of some integer. }\right\}
\end{gathered}
$$

Let $k$ be an integer and $\xi \in \mathfrak{g}^{(n)}$. Suppose we are given an operation $[\xi]_{k}$ on a space of functions on $\mathfrak{G}^{(n)} \times C^{m}$ such that

$$
\begin{aligned}
& f\left|[\xi \eta]_{k}=\left(f \mid[\xi]_{k}\right)\right|[\eta]_{k} \\
& f \mid[(1, \varphi)]_{k}=\varphi^{-k} f
\end{aligned}
$$


Let $\chi$ be a Dirichlet character modulo $N$ and let $H_{\chi}$ be a certain set of functions on $\mathfrak{h}^{(n)} \times \boldsymbol{C}^{m}$ such that

$$
f \mid[\xi]_{k}=\chi(\operatorname{det} D) f \quad \text { for any } \xi \in \Delta^{(n)}, P(\xi)=\left(\begin{array}{ll}
* & * \\
* & D
\end{array}\right) .
$$

For $\xi \in \bar{S}_{0}^{(n)}$ and $f \in H_{\chi}$, we set

$$
f\left|\left[\Delta^{(n)} \xi \Delta^{(n)}\right]_{k, \chi}=(\operatorname{det} P(\xi))^{(k / 2-(n+1)) / 2} \sum_{\nu} \chi\left(\operatorname{det} A_{\nu}\right) f\right|\left[\xi_{\nu}\right]_{k}
$$

where $\left\{\xi_{\nu}\right\}$ is a set of representatives of $\Delta^{(n)} \backslash \Delta^{(n)} \xi \Delta^{(n)}$ :

$$
\Delta^{(n)} \xi \Delta^{(n)}=\cup \Delta^{(n)} \xi_{\nu}, \quad P\left(\xi_{\nu}\right)=\left(\begin{array}{ll}
A_{\nu} & * \\
* & *
\end{array}\right) .
$$

(4.1) is independent on the choice of the representatives $\xi_{\nu}$.

We fix $\xi=(\alpha, \varphi) \in \bar{S}_{0}^{(n)}$. There exists a homomorphism $t_{\xi}$ of $\Gamma_{0}^{(n)}(N) \cap$ $\alpha^{-1} \Gamma_{0}^{(n)}(N) \alpha$ into $\boldsymbol{T}=\{z \in C|| z \mid=1\}$ such that

$$
L\left(\alpha \gamma \alpha^{-1}\right)=\xi L(\gamma) \xi^{-1}\left(1, t_{\xi}(\gamma)\right) .
$$

Then, by S. Hayakawa [3], the following assertions hold.

(i ) If $t_{\xi}^{k}$ is non-trivial on $\Gamma_{0}^{(n)}(N) \cap \alpha^{-1} \Gamma_{0}^{(n)}(N) \alpha$, then $f\left[\left[\Delta^{(n)} \xi \Delta^{(n)}\right]_{k, x}=0\right.$ for any $f \in H_{x}$.

(ii) The Hecke algebra $D\left(\Delta^{(n)}, \bar{S}^{(n)}\right)$ with respect to the Hecke pair $\left(\Delta^{(n)}, \bar{S}^{(n)}\right)$ is commutative and $D\left(\Delta^{(n)}, \bar{S}^{(n)}\right)=\otimes_{p \nmid N} D\left(\Delta^{(n)}, \bar{S}_{p}^{(n)}\right)$ where

$$
\bar{S}_{p}^{(n)}=\left\{\xi=(\alpha, \varphi) \in \bar{S}^{(n)} \mid \nu(\alpha)=p^{2 m}, m=0,1,2, \cdots\right\} .
$$

Furthermore if we put

$$
T_{p, i}^{(n)}=\Delta^{(n)}\left(\alpha_{p, i}, p^{i / 2}\right) \Delta^{(n)} \quad \text { with } \quad \alpha_{p, i}=\left(\begin{array}{ccc}
1_{i} & & \\
p 1_{n-1} & \\
& p^{2} 1_{i} & \\
& & p 1_{n-1}
\end{array}\right),
$$

$D\left(\Delta^{(n)}, \bar{S}^{(n)}\right)$ is generated by $T_{p, i}(0 \leqq i \leqq n)$ and $\left(1_{2 n}, t\right) \Delta^{(n)},(t \in T)$.

(iii) Let $n=2$ and $k$ an odd positive integer. We define an operation $[\xi]_{k}$ on a space of functions on $\mathfrak{h}^{(n)}$ by

$$
\left(f \mid[\xi]_{k}\right)(Z)=\varphi(Z)^{-k} f(\alpha(Z)) .
$$

and put $H_{\chi}=G_{k / 2}\left(\Gamma_{0}^{(2)}(N), \chi\right)$. For $f \in H_{\chi}$, we let

$$
f(Z)=\sum a(T) e(\operatorname{tr} T Z)
$$

and 


$$
\left(f \mid T_{p, i}\right)(Z)=\sum a_{p, i}(T) e(\operatorname{tr} T Z) .
$$

Then $a_{p, i}(T)$ are given by

$$
=\chi(p) p^{k / 2-3}\left\{\begin{array}{l}
a\left(T\left[\left(\begin{array}{ll}
1 & 0 \\
0 & p
\end{array}\right)\right]\right)+\sum_{s \in \bar{Z}_{p / p} Z} a\left(T\left[\left(\begin{array}{ll}
p & s \\
0 & 1
\end{array}\right)\right]\right)+\chi_{1}(p) p^{(k-3) / 2} h(T) a(T) \\
+\chi(p)^{2} p^{k-3}\left(a\left(T\left[\frac{1}{p}\left(\begin{array}{ll}
1 & 0 \\
0 & p
\end{array}\right)\right]\right)+\sum_{s \in \boldsymbol{Z} / p Z} a\left(T\left[\frac{1}{p}\left(\begin{array}{ll}
p & s \\
0 & 1
\end{array}\right)\right]\right)\right)
\end{array}\right\}
$$

where

$$
\chi_{1}(p)=\chi(p) \varepsilon_{p}^{k-1}
$$

and

$$
\begin{aligned}
& h(T)= \begin{cases}\left(\frac{\varepsilon}{p}\right) & \text { if } T \text { is equivalent to }\left(\begin{array}{ll}
\varepsilon & 0 \\
0 & 0
\end{array}\right) \text { with } \varepsilon \in(Z / p Z)^{\times} \text {over } Z_{p} / p Z_{p} \\
0 & \text { otherwise }\end{cases} \\
& \text { (4.4) } \quad a_{p, 2}(T)=a\left(p^{2} T\right) \\
& +\chi_{1}(p) p^{(k-5) / 2}\left\{g^{*}(T) a\left(T\left[\left(\begin{array}{ll}
1 & 0 \\
0 & p
\end{array}\right)\right]\right)+\sum_{s \in Z_{/ p} \boldsymbol{Z}} g\left(T\left[\left(\begin{array}{ll}
p & s \\
0 & 1
\end{array}\right)\right]\right) a\left(T\left[\left(\begin{array}{ll}
p & s \\
0 & 1
\end{array}\right)\right]\right)\right\} \\
& +\chi(p)^{2} p^{k-5} G(\operatorname{det} T) a(T) \\
& +\chi(p)^{2} p^{k-4}\left\{\sum_{s \in(\boldsymbol{Z} / p \boldsymbol{Z})^{\times}} a\left(T\left[\frac{1}{p}\left(\begin{array}{ll}
p & s \\
0 & p
\end{array}\right)\right]\right)+\sum_{s \in \sum_{\boldsymbol{Z} / p^{2} Z}} a\left(T\left[\frac{1}{p}\left(\begin{array}{ll}
p^{2} & s \\
0 & 1
\end{array}\right)\right]\right)\right. \\
& \left.+a\left(T\left[\frac{1}{p}\left(\begin{array}{ll}
1 & 0 \\
0 & p^{2}
\end{array}\right)\right]\right)\right\} \\
& +\chi(p)^{2} \chi_{1}(p) p^{(3 k-11) / 2}\left\{g(T) a\left(T\left[\frac{1}{p}\left(\begin{array}{ll}
1 & 0 \\
0 & p
\end{array}\right)\right]\right)+g^{*}(T) \sum_{s \in \boldsymbol{Z} / p \boldsymbol{Z}} a\left(T\left[\frac{1}{p}\left(\begin{array}{ll}
p & s \\
0 & 1
\end{array}\right)\right]\right)\right\} \\
& +\chi(p)^{4} p^{2 k-6} a\left(\frac{1}{p^{2}} T\right)
\end{aligned}
$$

where

$$
g *\left(\begin{array}{ll}
t_{1} & t_{2} \\
t_{2} & t_{3}
\end{array}\right)=\left(\frac{t_{1}}{p}\right), \quad g\left(\begin{array}{ll}
t_{1} & t_{2} \\
t_{2} & t_{3}
\end{array}\right)=\left(\frac{t_{3}}{p}\right)
$$

and

$$
G(T)= \begin{cases}p-1 & \text { if } t \equiv 0(\bmod p) \\ -1 & \text { if } t \neq \equiv 0(\bmod p)\end{cases}
$$

\section{$\S 5$. Definition of the Maass relation}

As before let $k$ be an odd positive integer and $m$ a positive integer. 
Let $\mathfrak{g}=\mathfrak{g}^{(1)}$ and $\xi=(\alpha, \varphi)$ an element of $\mathfrak{g}$ with $\alpha=\left(\begin{array}{ll}a & b \\ c & d\end{array}\right)$. We put $\ell=$ $\operatorname{det} \xi=\operatorname{det} \alpha$. We define an action $[\xi]_{k, m}$ on a function $\phi$ on $\mathfrak{h} \times \boldsymbol{C}$ by

$$
\left(\phi \mid[\xi]_{k, m}\right)(\tau, z)=\phi\left(\frac{a \tau+b}{c \tau+d}, \frac{\sqrt{\ell z}}{c \tau+d}\right) \varphi(\tau)^{-k} e\left(-\frac{m c z^{2}}{c \tau+d}\right) .
$$

It can be easily checked that

and

$$
\phi\left|[\xi \eta]_{k, m}=\left(\phi \mid[\xi]_{k, m}\right)\right|[\eta]_{k, m}
$$

$$
\phi \mid[(1, \varphi)]_{k, m}=\varphi(\tau)^{-k} \phi .
$$

We put $H_{\chi}=J_{k / 2, m}\left(\Gamma_{0}(N), \chi\right)$ this time. Note that $\phi \in J_{k / 2, m}\left(\Gamma_{0}(N), \chi\right)$ satisfies

$$
\phi \mid[\xi]_{k, m}=\chi(d) \phi
$$

for any $\xi \in \Delta_{0}(N)$. As in (4.1), we define a function $\phi \mid\left[\Delta_{0}(N) \xi \Delta_{0}(N)\right]_{0}$ by

$$
\phi\left|\left[\Delta_{0}(N) \xi \Delta_{0}(N)\right]_{0}=(\operatorname{det} \xi)^{k / 4-1} \sum_{\nu} \chi\left(a_{\nu}\right) \phi\right|\left[\xi_{\nu}\right]_{k, m}
$$

for a double coset $\Delta_{0}(N) \xi \Delta_{0}(N)=\cup \Delta_{0}(N) \xi_{\nu}, P\left(\xi_{\nu}\right)=\left(\begin{array}{ll}a_{\nu} & * \\ * & *\end{array}\right)$.

By (1.4) of G. Shimura [12], we have

$$
\phi \mid\left[\Delta_{0}(N) \xi \Delta_{0}(N)\right]_{0}=0
$$

if $\operatorname{det} \xi$ is not a square, i.e. $\xi \in \bar{S}_{0}-\bar{S}$. Finally we define the Hecke operator on $J_{k / 2, m}\left(\Gamma_{0}(N), \chi\right)$ by

$$
\left(\phi \mid\left[\Delta_{0}(N) \xi \Delta_{0}(N)\right]\right)(\tau, z)=\left(\phi \mid\left[\Delta_{0}(N) \xi \Delta_{0}(N)\right]_{0}\right)(\tau, \sqrt{\ell} z) .
$$

Proposition 2. Let $\xi$ be an element of $\bar{S}$ and $\ell=\operatorname{det} \xi$. Then the Hecke operator $\left[\Delta_{0}(N) \xi \Delta_{0}(N)\right]$ is a linear mapping from $J_{k / 2, m}\left(\Gamma_{0}(N), \chi\right)$ to $J_{k / 2, m \varepsilon}\left(\Gamma_{0}(N), \chi\right)$.

It is easy to prove this by direct calculations.

Let $f \in G_{k / 2}\left(\Gamma_{0}^{(2)}(N), \chi\right)$ be a Siegel modular form and $\phi_{m, f}(\tau, z)$ the Jacobi form of index $m$ obtained from $f$ by Proposition 1 . We set the following definition.

Definition 2. Let $p$ be a prime number such that $(p, N)=1$. Put

$$
\xi_{p}=\left(\left(\begin{array}{cc}
1 & 0 \\
0 & p^{2}
\end{array}\right), p^{1 / 2}\right)
$$


and

$$
\eta_{p}=\left(\left(\begin{array}{ll}
p & 0 \\
0 & p
\end{array}\right), 1\right) .
$$

A Siegel modular form $f$ is said to satisfy the Maass relation for $p$ if and only if

$$
\begin{aligned}
\phi_{m p^{2}, f}= & \phi_{m, f}\left|\left[\Delta_{0}(N) \xi_{p} \Delta_{0}(N)\right]-\varepsilon_{p}^{k-1}\left(\frac{m}{p}\right) p^{1 / 2} \phi_{m, f}\right|\left[\Delta_{0}(N) \eta_{p} \Delta_{0}(N)\right] \\
& -p^{2} \phi_{m / p^{2}, f} \mid\left[\Delta_{0}(N) \eta_{p} \Delta_{0}(N)\right]^{2}
\end{aligned}
$$

for any $m$.

We regard that if $m$ is not an integer, $\phi_{m, f}(\tau, z)$ is identically zero.

For later use, we rewrite (5.1) by the Fourier coefficients of $f$. As is well known, a complete set of representatives of $\Delta_{0}(N) \backslash \Delta_{0}(N) \xi_{p} \Delta_{0}(N)$ is given by

$$
\begin{aligned}
& \alpha_{b}^{*}=\left(\left(\begin{array}{cc}
1 & b \\
0 & p^{2}
\end{array}\right), p^{1 / 2}\right) \quad 0 \leqq b<p^{2}, \\
& \beta_{h}^{*}=\left(\left(\begin{array}{cc}
p & h \\
0 & p
\end{array}\right), \varepsilon_{p}\left(\frac{h}{p}\right)\right) \quad 0<h<p, \\
& \sigma^{*}=\left(\left(\begin{array}{cc}
p^{2} & 0 \\
0 & 1
\end{array}\right), p^{-1 / 2}\right)
\end{aligned}
$$

(cf. G. Shimura [12]). By Proposition 1, $\phi_{m, f}(\tau, z)$ has a Fourier expansion

$$
\phi_{m, f}(\tau, z)=\sum_{\substack{n \geqq 0, r \\ 4 n m \geqq r^{2}}} a(n, r, m) e(n \tau+r z) .
$$

Therefore, by the definition of Hecke operators, we obtain

$$
\begin{aligned}
\left(\phi_{m, f} \mid\left[\Delta_{0}(N) \xi_{p} \Delta_{0}(N)\right]\right)(\tau, z) & \\
= & p^{k / 2-2}\left\{p^{-k / 2} \sum_{b} \phi_{m, f}\left(\frac{\tau+b}{p^{2}}, z\right)+\varepsilon_{p}^{k} \chi(p) \sum_{h} \phi_{m, f}\left(\tau+\frac{h}{p}, p z\right)\left(\frac{-h}{p}\right)\right. \\
& \left.+\chi(p)^{2} p^{k / 2} \phi_{m, f}\left(p^{2} \tau, p^{2} z\right)\right\} .
\end{aligned}
$$

By an elementary calculation and the classical knowledge on the Gauss sum:

$$
\sum_{0<h<p} e\left(\frac{n h}{p}\right)\left(\frac{-h}{p}\right)=\varepsilon_{p}^{-1}\left(\frac{n}{p}\right) p^{1 / 2}
$$

we have 


$$
\begin{aligned}
& \left(\phi_{m, f} \mid\left[\Delta_{0}(N) \xi_{p} \Delta_{0}(N)\right]\right)(\tau, z) \\
& =\sum_{\substack{n, r \\
4 n p^{2} m \geqq r^{2}}} a\left(n p^{2}, r, m\right) e(n \tau+r z) \\
& \quad+\chi(p) \varepsilon_{p}^{k-1} p^{(k-3) / 2} \sum_{\substack{n, r \\
4 n m \geqq r^{2}}}\left(\frac{n}{p}\right) a(n, r, m) e(n \tau+r p z) \\
& \quad+\chi(p)^{2} p^{k-2} \sum_{\substack{n, r \\
4 n m \geqq r^{2}}} a(n, r, m) e\left(n p^{2} \tau+r p^{2} z\right) .
\end{aligned}
$$

On the other hand, we can easily see that

$$
\left(\phi_{m, f} \mid\left[\Delta_{0}(N) \eta_{p} \Delta_{0}(N)\right]\right)(\tau, z)=p^{k / 2-2} \chi(p) \sum_{\substack{n, r \\ 4 n m \geqq r^{2}}} a(n, r, m) e(n \tau+r p z) .
$$

Hence the Maass relation (5.1) is equivalent to

$$
\begin{aligned}
a\left(n, r, m p^{2}\right)= & a\left(n p^{2}, r, m\right)+\chi(p) \varepsilon_{p}^{k-1} p^{(k-3) / 2}\left(\left(\frac{n}{p}\right)-\left(\frac{m}{p}\right)\right) a\left(n, \frac{r}{p}, m\right) \\
& +\chi(p)^{2} p^{k-2}\left(a\left(\frac{n}{p^{2}}, \frac{r}{p^{2}}, m\right)-a\left(n, \frac{r}{p^{2}}, \frac{m}{p^{2}}\right)\right) .
\end{aligned}
$$

In particular, if $p$ divides $n$ and $m$, then

$$
\begin{aligned}
& a\left(n, r, m p^{2}\right)-\chi(p)^{2} p^{k-2} a\left(\frac{n}{p^{2}}, \frac{r}{p^{2}}, m\right) \\
& \quad=a\left(n p^{2}, r, m\right)-\chi(p)^{2} p^{k-2} a\left(n, \frac{r}{p^{2}}, \frac{m}{p^{2}}\right) .
\end{aligned}
$$

And if $p$ does not divide $m$, then

$$
\begin{aligned}
a\left(n, r, m p^{2}\right)= & a\left(n p^{2}, r, m\right)+\chi(p) \varepsilon_{p}^{k-1} p^{(k-3) / 2}\left(\left(\frac{n}{p}\right)-\left(\frac{m}{p}\right)\right) a\left(n, \frac{r}{p}, m\right) \\
& +\chi(p)^{2} p^{k-2} a\left(\frac{n}{p^{2}}, \frac{r}{p^{2}}, m\right) .
\end{aligned}
$$

It can be shown by using the formula of local densities of quadratic forms that the Eisenstein series satisfies the above Maass relation (Y. Kitaoka [7]).

\section{§6. Dirichlet series}

In this section we consider two kinds of Dirichlet series as stated in the introduction. We always assume that $p$ is a prime number such that $(p, N)=1$. 
Theorem 3. Let $f$ be a Siegel modular form on $\Gamma_{0}^{(2)}(N)$ of weight $k / 2$ and characier $\chi$, and let

$$
\phi_{1, f}(\tau, z)=c_{0, f}(\tau) \Theta_{0}(\tau, z)+c_{1 / 2, f}(\tau) \Theta_{1 / 2}(\tau, z)
$$

be the Jacobi form of index 1 obtained from $f$. Put

$$
c_{0, f}(\tau)=\sum_{n \geqq 0} \gamma(n) e(n \tau) \text {. }
$$

Suppose that $f$ is an eigenform of $T_{p, 1}$ with an eigenvalue $\lambda_{1}(p)$ and satisfies the Maass relation for $p$. Then we have

$$
\omega(p) \gamma(n)=\gamma\left(n p^{2}\right)+\chi(p) \varepsilon_{p}^{k-1} p^{(k-3) / 2}\left(h_{p}(n)-1\right) \gamma(n)+\chi(p)^{2} p^{k-3} \gamma\left(\frac{n}{p^{2}}\right)
$$

where

$$
\omega(p)=\frac{\chi(p)^{-1} p^{3-k / 2}}{p+1} \lambda_{1}(p)
$$

and

$$
h_{p}(n)= \begin{cases}0 & \text { if } p \nmid n \\ 1 & \text { if } p \mid n\end{cases}
$$

Proof. By assumption, $f \mid T_{p, 1}=\lambda_{1}(p) f$. If we put $T=\left(\begin{array}{ll}n & 0 \\ 0 & 1\end{array}\right)$ in (4.3), we have

$$
\begin{aligned}
& \quad \chi(p)^{-1} p^{3-k / 2} \lambda_{1}(p) a(n, 0,1) \\
& \quad=a\left(n, 0, p^{2}\right)+\sum_{s \in \mathbf{Z} / p Z} a\left(n p^{2}, 2 n p s, n s^{2}+1\right)+\chi(p) \varepsilon_{p}^{k-1} p^{(k-3) / 2} h_{p}(n) a(n, 0,1) \\
& \quad+\chi(p)^{2} p^{k-3}\left(a\left(\frac{n}{p^{2}}, 0,1\right)+\sum_{s \in \sum_{/ p Z}} a\left(n, \frac{2 n s}{p}, \frac{n s^{2}+1}{p^{2}}\right)\right) .
\end{aligned}
$$

We note that $a\left(n, 2 n s / p,\left(n s^{2}+1\right) / p^{2}\right)=0$ for all $s \in Z / p Z$. Let us consider the second term of the right hand side. When $n$ is prime to $p$, we have, by (5.4),

$$
\begin{aligned}
& a\left(n p^{2}, 2 n p s, n s^{2}+1\right)=a\left(n s^{2}+1,2 n p s, n p^{2}\right) \\
&= a\left(\left(n s^{2}+1\right) p^{2}, 2 n p s, n\right) \\
&+\chi(p) \varepsilon_{p}^{k-1} p^{(k-3) / 2}\left(\left(\frac{n s^{2}+1}{p}\right)-\left(\frac{n}{p}\right)\right) a\left(n s^{2}+1,2 n s, n\right) \\
&+\chi(p)^{2} p^{k-2} a\left(\frac{n s^{2}+1}{p^{2}}, \frac{2 n s}{p}, n\right) \\
&= a\left(n, 0, p^{2}\right)+\chi(p) \varepsilon_{p}^{k-1} p^{(k-3) / 2}\left(\left(\frac{n s^{2}+1}{p}\right)-\left(\frac{n}{p}\right)\right) a(n, 0,1) .
\end{aligned}
$$


On the other hand, when $p$ divides $n$, we regard $n s^{2}+1$ as $m$ in (5.4) to obtain

$$
\begin{aligned}
a\left(n p^{2},\right. & \left.2 n p s, n s^{2}+1\right) \\
= & a\left(n, 2 n p s,\left(n s^{2}+1\right) p^{2}\right) \\
& -\chi(p) \varepsilon_{p}^{k-1} p^{(k-1) / 2}\left(\left(\frac{n}{p}\right)-\left(\frac{n s^{2}+1}{p}\right)\right) a\left(n, 2 n s, n s^{2}+1\right) \\
& -\chi(p)^{2} p^{k-2} a\left(\frac{n}{p^{2}}, \frac{2 n s}{p}, n s^{2}+1\right) \\
= & a\left(n, 0, p^{2}\right)+\chi(p) \varepsilon_{p}^{k-1} p^{(k-3) / 2}\left(\left(\frac{n s^{2}+1}{p}\right)-\left(\frac{n}{p}\right)\right) a(n, 0,1) \\
& -\chi(p)^{2} p^{k-2} a\left(\frac{n}{p^{2}}, 0,1\right) .
\end{aligned}
$$

But the expression (6.5) is the same as (6.6), since $a\left(n / p^{2}, 0,1\right)=0$, when $n$ is prime to $p$. We again use (5.4) for $a\left(n, 0, p^{2}\right)$ and get $a\left(n, 0, p^{2}\right)$

$$
=a\left(n p^{2}, 0,1\right)+\chi(p) \varepsilon_{p}^{k-1} p^{(k-3) / 2}\left(\left(\frac{n}{p}\right)-1\right) a(n, 0,1)+\chi(p)^{2} p^{k-2} a\left(\frac{n}{p^{2}}, 0,1\right) .
$$

By an elementary calculation, we have

$$
\sum_{s \in Z / p Z Z}\left(\frac{n s^{2}+1}{p}\right)-p\left(\frac{n}{p}\right)=\left\{\begin{array}{cl}
-(p+1)\left(\frac{n}{p}\right) & \text { if } p \nmid n \\
p & \text { if } p \mid n .
\end{array}\right.
$$

Hence

$$
\begin{aligned}
& \chi(p)^{-1} p^{3-k / 2} \lambda_{1}(p) a(n, 0,1) \\
&=(p+1)\left\{a\left(n p^{2}, 0,1\right)+\chi(p) \varepsilon_{p}^{k-1} p^{(k-3) / 2}\left(h_{p}(n)-1\right) a(n, 0,1)\right. \\
&\left.+\chi(p)^{2} p^{k-3} a\left(\frac{n}{p^{2}}, 0,1\right)\right\} .
\end{aligned}
$$

This concludes the proof, because $\gamma(n)=a(n, 0,1)$.

CoRollary 1. Let the notation and the assumptions be as in Theorem 3. Let $T(n)$ be a Hecke operator acting on $G_{(k-1) / 2}\left(\Gamma_{0}(N), \chi_{1}\right)$. Then the form $c_{0, f}(\tau)$ is an eigenform of $T\left(p^{2}\right)$ with an eigenvalue $\omega(p)+\chi_{1}(p) p^{(k-3) / 2}$.

Proof. In general, let $g(\tau)$ be an elliptic modular form on $\Gamma_{0}(M)$ of 
integral weight $m$ and character $\psi$. If we put $g(\tau)=\sum a(n) e(n \tau)$ and $\left(g \mid T\left(p^{2}\right)\right)(\tau)=\sum a^{\prime}(n) e(n \tau)$, it is well known that

$$
a^{\prime}(n)=a\left(n p^{2}\right)+\psi(p) p^{m-1} h_{p}(n) a(n)+\psi(p)^{2} p^{2(m-1)} a\left(\frac{n}{p^{2}}\right)
$$

for $p$ such that $(p, M)=1$. Hence we get our assersion from (6.1).

We get the following proposition by the same method as in G. Shimura [12].

Proposition 3. Let the notation be as in Theorem 3. Suppose that the assumptions of the theorem are valid for all $p$ such that $(p, N)=1$. Let $t$ be a positive integer which has no square factor and is prime to $N$. Then

$$
\sum_{(n, N)=1} \gamma\left(t n^{2}\right) n^{-s}=\gamma(t) \prod_{p \nmid N} \frac{1+\left(1-h_{p}(t)\right) \chi_{1}(p) p^{(k-3) / 2-s}}{1-\omega(p) p^{-s}+\chi(p)^{2} p^{k-3-2 s}} .
$$

Let $g(\tau)$ be an elliptic modular form on $\Gamma_{0}(M)$ of the (integral) weight $m$ and character $\psi$. We assume that $g(\tau)$ is a common eigenform of all the Hecke operators $T\left(p^{2}\right)$ for $p$ such that $(p, M)=1$, with the eigenvalue $\mu\left(p^{2}\right)$. Then the formal Dirichlet series $\sum_{(n, M)=1} \mu\left(n^{2}\right) n^{-s}$ has the following Euler product:

$$
\sum_{(n, M)=1} \mu\left(n^{2}\right) n^{-s}=\sum_{p \nmid M}\left(1-\left(\mu\left(p^{2}\right)-p^{m-1} \psi(p)\right) p^{-s}+p^{2 m-2} \psi(p)^{2} p^{-2 s}\right)^{-1} .
$$

In fact, this follows from the relation of the Hecke operators:

$$
\left(T\left(p^{2}\right)-p T(p, p)\right) T\left(p^{2 e}\right)=T\left(p^{2(e+1)}\right)+p^{2} T(p, p)^{2} T\left(p^{2(e-1)}\right) .
$$

Let us define the Dirichlet series $L_{2}(s, g)$ by the left hand side of (6.8):

$$
L_{2}(s, g)=\sum_{(n, M)=1} \mu\left(n^{2}\right) n^{-s} .
$$

Remark. We also get a similar results for $c_{1 / 2, f}(\tau)$. Put $T=\left(\begin{array}{cc}n & 1 / 2 \\ 1 / 2 & 1\end{array}\right)$ in (4.3). Then we get this time

$$
\begin{aligned}
\omega(p) a(n, 1,1)= & a\left(\frac{p^{2}(4 n-1)+1}{4}, 1,1\right) \\
& +\chi(p) \varepsilon_{p}^{k-1} p^{(k-3) / 2}\left(\left(\frac{n}{p}\right)-1+\left(\frac{n}{p}\right) \kappa_{p}(n)\right) a(n, 1,1) \\
& +\chi(p)^{2} p^{k-3} a\left(\frac{(4 n-1) / p^{2}+1}{4}, 1,1\right)
\end{aligned}
$$


where $\omega(p)$ is the same as in Theorem 3 and $\kappa_{p}(n)$ is defined by

$$
\kappa_{p}(n)=\left\{\begin{array}{cl}
-1 & \text { if } p \nmid n, p \nmid 4 n-1 \\
0 & \text { otherwise }
\end{array}\right.
$$

Let $c_{1 / 2, f}(\tau)=\sum \gamma_{1}(n) e((n / 4) \tau)$ be its Fourier expansion. Then Fourier coefficients are given by

$$
\gamma_{1}(n)= \begin{cases}a\left(\frac{n+1}{4}, 1,1\right) & \text { if } n \equiv 3(\bmod 4) \\ 0 & \text { otherwise } .\end{cases}
$$

Hence (6.9) is equivalent to

$$
\begin{aligned}
& \omega(p) \gamma_{1}(n) \\
& =\gamma_{1}\left(p^{2} n\right)+\chi(p) \varepsilon_{p}^{k-1} p^{(k-3) / 2}\left(\left(\frac{(n+1) / 4}{p}\right)-1+\left(\frac{(n+1) / 4}{p}\right) \kappa_{p}\left(\frac{n+1}{4}\right)\right) \gamma_{1}(n) \\
& \quad+\chi(p)^{2} p^{k-3} \gamma_{1}\left(\frac{n}{p^{2}}\right) .
\end{aligned}
$$

But it is easily seen that

$$
\left(\frac{(n+1) / 4}{p}\right)\left(1+\kappa_{p}\left(\frac{n+1}{4}\right)\right)=h_{p}(n)= \begin{cases}1 & \text { if } p \mid n \\ 0 & \text { otherwise }\end{cases}
$$

for all $n$ such that $n \equiv 3(\bmod 4)$. Therefore, we have

$$
\sum_{(n, N)=1} \gamma_{1}\left(t n^{2}\right) n^{-s}=\gamma_{1}(t) \prod_{p \nmid N} \frac{1+\left(1-h_{p}(t)\right) \chi_{1}(p) p^{(k-3) / 2-s}}{1-\omega(p) p^{-s}+\chi(p)^{2} p^{k-3-2 s}}
$$

where $t$ is a square free integer such that $(t, N)=1$ and $t \equiv 3(\bmod 4)$.

THEOREM 4. Let $f$ be a common eigenform of $T_{p, 1}$ and $T_{p, 2}$ for all $p$. Suppose further that $f$ satisfies the Maass relations for all $p$. Let $\mathscr{R}=$ $\left\{M \in M_{2}(Z) \cap G L(2, Q) \mid(\operatorname{det} M, N)=1\right\} / G L(2, Z)$. Then

$$
\sum_{M \in \mathscr{R}} a(T[M])|\operatorname{det} M|^{-s}=L_{2}\left(s, c_{0, f}\right) L_{2}\left(s+1, c_{0, f}\right) \prod_{p \nmid N} P_{p}\left(p^{-s} ; T\right)
$$

where $P_{p}\left(p^{-s} ; T\right)$ is a polynomial of $p^{-s}$ of degree at most 3 .

Proof. We quote a result of T. Ibukiyama [5]. He showed that, if $f$ is a common eigenform of $T_{p, 1}$ and $T_{p, 2}$ with eigenvalues $\lambda_{1}(p)$ and $\lambda_{2}(p)$ respectively, then the Dirichlet series $\sum_{M \in \mathscr{R}} a(T[M])|\operatorname{det} M|^{-s}$ equals to

$$
\prod_{p \nmid N} \frac{P_{p}\left(p^{-s} ; T\right)}{Q_{p}\left(p^{-s}\right)}
$$


where $P_{p}\left(p^{-s} ; T\right)$ and $Q_{p}\left(p^{-s}\right)$ are polynomials of $p^{-s}$ and

$$
\begin{aligned}
& \operatorname{deg} P_{p}(x ; T) \leqq 3 \\
& \begin{aligned}
Q_{p}(x)= & 1-\chi(p)^{-1} p^{3-k / 2} \lambda_{1}(p) x+\left(p \lambda_{2}(p)+\chi(p)^{2} p^{k-4}\left(1+p^{2}\right)\right) x^{2} \\
& -\chi(p) p^{k / 2+1} \lambda_{1}(p) x^{3}+\chi(p)^{4} p^{2 k-4} x^{4}
\end{aligned}
\end{aligned}
$$

Now we suppose further that $f$ satisfies the Maass relation. Then it is easily seen that

$$
\lambda_{2}(p)=\omega(p)^{2}+\chi(p)^{2} p^{k-5}\left(p^{2}+1\right)(p-1) .
$$

Therefore $Q_{p}(x)$ decomposes into

$$
\left(1-\omega(p) x+\chi(p)^{2} p^{k-3} x^{2}\right)\left(1-\omega(p) p x+\chi(p)^{2} p^{k-1} x^{2}\right) .
$$

Hence we get our assertion.

Next we consider another type of Dirichlet series. In [4], T. Hina showed that $\sum_{\nu=0}^{\infty} a\left(p^{2 \nu} T\right) x^{\nu}$ is a rational function of $x$, and its denominator and numerator have degree 4 and at most 3 respectively. We show that, for a Maass form, the above Dirichlet series has the denominator of degree 3.

Lemma 5. Let $f$ be an eigenform of $T_{p, 1}$, which satisfies the Maass relation for $p$. Let $T=\left(\begin{array}{cc}n & r / 2 \\ r / 2 & m\end{array}\right)$ be an element of $\Lambda_{2}$ such that $m \neq \equiv 0(\bmod$ p). Then

$$
\begin{aligned}
& \sum_{\nu=0}^{\infty} a\left(p^{2 \nu} n, p^{\nu} r, m\right) x^{\nu} \\
= & \frac{a(n, r, m)+\left(a\left(p^{2} n, p r, m\right)-\omega(p) a(n, r, m)\right) x}{1-\omega(p) x+\chi(p)^{2} p^{k-3} x^{2}}
\end{aligned}
$$

where $\omega(p)$ is defined by (6.2).

Proof. We have already known the expression (6.11) for $T=\left(\begin{array}{ll}n & 0 \\ 0 & 1\end{array}\right)$ by Proposition 3. Let $T=\left(\begin{array}{cc}n & r / 2 \\ r / 2 & m\end{array}\right)$ with $m \neq 0(\bmod p)$. We put

$$
H(x ; T)=\sum_{\nu=0}^{\infty} a\left(p^{2 \nu} n, p^{\nu} r, m\right) x^{\nu} .
$$

Then the coefficient of $x^{\nu}$ for $\nu \geqq 2$ in

$$
\left(1-\omega(p) x+\chi(p)^{2} p^{k-3} x^{2}\right) H(x ; T)
$$


is

$$
a\left(p^{2 \nu} n, p^{\nu} r, m\right)-\omega(p) a\left(p^{2(\nu-1)} n, p^{\nu-1} r, m\right)+\chi(p)^{2} p^{k-3} a\left(p^{2(\nu-2)} n, p^{\nu-2} r, m\right) .
$$

Therefore it suffices to show that (6.13) is identically zero. Put

$$
T=\left(\begin{array}{cc}
p^{2(\nu-1)} n, \frac{p^{\nu-1} r}{2} \\
\frac{p^{\nu-1} r}{2}, & m
\end{array}\right)
$$

in (4.3). Since $m \neq \equiv 0(\bmod p)$, we have

$$
\begin{aligned}
& \chi(p)^{-1} p^{3-k / 2} \lambda_{1}(p) a\left(p^{2(\nu-1)} n, p^{\nu-1} r, m\right) \\
& =a\left(p^{2(\nu-1)} n, p^{\nu} r, m\right)+\sum_{s \in Z / p Z} a\left(p^{2 \nu} n, 2 p^{2 \nu-1} s n+p^{\nu} r, p^{2(\nu-1)} s^{2} n+p^{\nu-1} s r+m\right) \\
& \quad+\chi(p) \varepsilon_{p}^{k-1} p^{(k-3) / 2}\left(\frac{m}{p}\right) a\left(p^{2(\nu-1)} n, p^{\nu-1} r, m\right)+\chi(p)^{2} p^{k-3} a\left(p^{2 \nu-4} n, p^{\nu-2} r, m\right) .
\end{aligned}
$$

But

$$
\begin{aligned}
a\left(p^{2 \nu} n, 2 p^{2 \nu-1} s n+p^{\nu} r, p^{2(\nu-1)} s^{2} n+p^{\nu-1} s r+m\right) \\
=a\left(p^{2 \nu-2} n, p^{\nu} r, p^{2} m\right)+\chi(p) \varepsilon_{p}^{k-1} p^{(k-3) / 2}\left(\frac{m}{p}\right) a\left(p^{2 \nu-2} n, p^{\nu-1} r, m\right) \\
\quad-\chi(p)^{2} p^{k-2} a\left(p^{2 \nu-4} n, p^{\nu-2} r, m\right) .
\end{aligned}
$$

Therefore

$$
\begin{aligned}
& \omega(p) a\left(p^{2(\nu-1)} n, p^{\nu-1} r, m\right) \\
&= a\left(p^{2 \nu-2} n, p^{\nu} r, p^{2} m\right)+\chi(p) \varepsilon_{p}^{k-1} p^{(k-3) / 2}\left(\frac{m}{p}\right) a\left(p^{2 \nu-2} n, p^{\nu-1} r, m\right) \\
&-\chi(p)^{2} p^{k-3}(p-1) a\left(p^{2 \nu-4} n, p^{\nu-2} r, m\right) \\
&= a\left(p^{2 \nu} n, p^{\nu} r, m\right)+\chi(p)^{2} p^{k-3} a\left(p^{2 \nu-4} n, p^{\nu-2} r, m\right) .
\end{aligned}
$$

This is what we wanted.

TheOREM 5. Let $f(Z)$ be an eigenform of $T_{p, 1}$, which satisfies the Maass relation for $p$. Let $T$ be an element of $\Lambda_{2}$ such that $T \neq 0(\bmod p)$ and let $d$ be a non-negative integer. Then

$$
\sum_{\nu=0}^{\infty} a\left(p^{2 \nu+2 d} T\right) x^{\nu}=\frac{R_{p}(x ; d, T)}{\left(1-\chi(p)^{2} p^{k-2} x\right)\left(1-\left(\omega(p)^{2}-2 \chi(p)^{2} p^{k-3}\right) x+\chi(p)^{4} p^{2(k-3)} x^{2}\right)}
$$

where $R_{p}(x ; d, T)$ is a polynomial of $x$ such that $\operatorname{deg}\left(R_{p}(x ; d, T)\right) \leqq 2$. 
Proof. We may assume that $m \neq \equiv 0(\bmod p)$, because $a(T)=a(T[U])$ for any $U \in G L(2, Z)$. We put

$$
K(x ; d, T)=\left(1-\chi(p)^{2} p^{k-2} x\right) \times \sum_{\nu=0}^{\infty} a\left(p^{2 \nu+2 d} T\right) x^{\nu}
$$

By using (5.3) (5.4) successively, we get

$$
\begin{aligned}
K(x ; d, T)= & a\left(p^{2 d} T\right)+\sum_{\nu=1}^{\infty}\left(a\left(p^{4 \nu-2+4 d} n, p^{2 \nu+2 d} r, p^{2} m\right)\right. \\
& \left.-\chi(p)^{2} p^{k-2} a\left(p^{4 \nu-4+4 d} n, p^{2 \nu-2+2 d} r, m\right)\right) x^{\nu} \\
= & a\left(p^{2 d} T\right)+\sum_{\nu=1}^{\infty}\left(a\left(p^{4 \nu+4 d} n, p^{2 \nu+2 d} r, m\right)\right. \\
& \left.-\chi(p) \varepsilon_{p}^{k-1} p^{(k-3) / 2}\left(\frac{m}{p}\right) a\left(p^{4 \nu-2+4 d} n, p^{2 \nu-1+2 d} r, m\right)\right) x^{\nu} .
\end{aligned}
$$

Put

$$
K_{1}(x ; T)=K_{1}(x ; n, r, m)=\sum_{\nu=0}^{\infty} a\left(p^{4 \nu} n, p^{2 \nu} r, m\right) x^{\nu}
$$

By Lemma 5, we have

$$
\begin{aligned}
& K_{1}\left(x^{2} ; T\right)=\frac{1}{2}(H(x ; T)+H(-x ; T)) \\
& =\frac{a(n, r, m)+\left[a(n, r, m) \chi(p)^{2} p^{k-3}+\omega(p)\left(a\left(p^{2} n, p r, m\right)-\omega(p) a(n, r, m)\right)\right] x^{2}}{1-\left(\omega(p)^{2}-2 \chi(p)^{2} p^{k-3}\right) x^{2}+\chi(p)^{4} p^{2(k-3)} x^{4}}
\end{aligned}
$$

Since

$$
\begin{aligned}
K(x ; d, T)= & a\left(p^{2 d} n, p^{2 d} r, p^{2 d} m\right)-a\left(p^{4 d} n, p^{2 d} r, m\right)+K_{1}\left(x ; p^{4 d} n, p^{2 d} r, m\right) \\
& -\chi(p) \varepsilon_{p}^{k-1} p^{(k-3) / 2}\left(\frac{m}{p}\right) K_{1}\left(x ; p^{4 d+2} n, p^{2 d+1} r, m\right) x
\end{aligned}
$$

its denominator is equal to

$$
1-\left(\omega(p)^{2}-2 \chi(p)^{2} p^{k-3}\right) x+\chi(p)^{4} p^{2(k-3)} x^{2} .
$$

By comparing the Hina's result, there is one cancellation of a linear factor, so we get the theorem.

\section{§7. Remark}

We can ask the following questions. 
1. What is the Maass relation for $p=2$ ?

2. What is the relation between the Jacobi forms of index 1 and $p$ ? Eisenstein series is a typical example. 1 is not yet solved. For 2, let $E(z, s)=\sum a(T) e(\operatorname{tr} T Z)$. If $n$ and $m$ are integers prime to $p$, then

$$
\begin{aligned}
a\left(\begin{array}{cc}
n p^{\ell} & 0 \\
0 & m p
\end{array}\right)= & a\left(\begin{array}{cc}
n p^{\ell+1} & 0 \\
0 & m
\end{array}\right)+\chi(p) \varepsilon_{p}^{k-1}\left(\left(\frac{n}{p}\right)-\left(\frac{m}{p}\right)\right) a\left(\begin{array}{cc}
n p^{\ell} & 0 \\
0 & m
\end{array}\right) \\
& -\chi(p) \varepsilon_{p}^{k-1}\left(\frac{n}{p}\right) p^{(k-3) / 2} a\left(\begin{array}{cc}
n p^{\ell-1} & 0 \\
0 & m
\end{array}\right) \quad \text { if } \ell \text { is even } \\
a\left(\begin{array}{cc}
n p^{\ell} & 0 \\
0 & m p
\end{array}\right)= & a\left(\begin{array}{cc}
n p^{\ell+1} & 0 \\
0 & m
\end{array}\right)+\chi(p) \varepsilon_{p}^{k-1}\left(\left(\frac{-n}{p}\right)-\left(\frac{m}{p}\right)\right) a\left(\begin{array}{cc}
n p^{\ell} & 0 \\
0 & m
\end{array}\right) \\
& -\chi(p) \varepsilon_{p}^{k-1}\left(\frac{-n}{p}\right) p^{(k-3) / 2} a\left(\begin{array}{cc}
n p^{\ell-1} & 0 \\
0 & m
\end{array}\right) \quad \text { if } \ell \text { is odd }
\end{aligned}
$$

(cf. Y. Kitaoka [7]). It is natural that we also call these ones the Maass relations.

Recently I was informed by T. Ibukiyama and S. Hayakawa that V. G. Juravlev obtained similar results on Siegel modular forms of half integral weight.

\section{REFERENCES}

[1] A. N. Andrianov, Modular descent and the Saito-Kurokawa conjecture, Invent. Math., 53 (1979), 267-280.

[2] M. Eichler and D. Zagier, The theory of Jacobi forms, Progress in Mathematics, 55, Birkhäuser, 1985.

[ 3 ] S. Hayakawa, On Hecke operators of Siegel modular forms of half integral weight, preprint.

[ 4 ] T. Hina, On Siegel modular forms of second degree which has half integral weight, (in Japanese), preprint.

[5] T. Ibukiyama, On construction of half integral weight Siegel modular forms of $S p(2, \boldsymbol{R})$ from automorphic forms of the compact twist $S p(2)$, MPI/SFB 84-18.

[6] J. Igusa, Theta Functions, Die Grundlehren der Mathematischen Wissenschaften in Einzeldarstellungen, 194, Springer-Verlag, Berlin 1972.

[ 7 ] Y. Kitaoka, A note on local densities of quadratic forms, Nagoya Math. J., 92 (1983), 145-152.

[ 8 ] H. Maass, Über eine Spezialschar von Modulformen zweiten Grades, Invent. Math., 52 (1979), 95-104.

[ 9 ] — U Über eine Spezialschar von Modulformen zweiten Grades II, Invent. Math., 53 (1979), 249-253.

[10] — Über eine Spezialschar von Modulformen zweiten Grades III, Invent. Math., 53 (1979), 255-265.

[11] O. T. O'Meara, Introduction to quadratic forms, Die Grundlehren der Mathematischen Wissenschaften in Einzeldarstellungen, 117, Springer-Verlag 1963. 
[12] G. Shimura, On modular forms of half integral weight, Ann. of Math., 97 (1973), $440-481$.

[13] D. Zagier, Sur la conjecture de Saito-Kurokawa (d'apres H. Maass), Seminaire Delange-Pisot-Poitou, 1979-1980, in Progress in Math. 12, Birkhäuser-Verlag, 1980, 371-394.

Department of Mathematics

Faculty of Science

Nagoya University

Chikusa-ku, Nagoya 464

Japan 